\title{
Rotational Invariance in Visual Pattern Recognition by Pigeons and Humans
}

\author{
Valerie D. Hollard and Juan D. Delius
}




\section{Rotational Invariance in Visual Pattern Recognition by Pigeons and Humans}

Abstract. Pigeons and humans chose which one of two alternative visual forms was identical to, or a mirror image of, a previously presented sample form. The two comparison forms were presented in various orientations with respect to the sample. The two species yielded similar accuracies, but although human reaction times depended linearly on the angular disparities, those of the pigeon did not. Humans appeared to apply a well-known, thoughtlike, mental rotation procedure to the problem, whereas pigeons seemed to rely on a more efficient automatic process that humans can use only in simpler rotational invariance tasks. Mirror-image forms may be better discriminated by the pigeon's visual system than by the human one.

The visual recognition of objects regardless of their relative spatial orientation is a competence that humans use constantly. Research on this capability has accordingly a long history $(I)$, and individual proficiency in it is assessed by several intelligence and aptitude tests (2). Certain feats of rotational invariance are believed to implicate cognitive skills and to involve mental imagery (3). Although casual observations of higher animals dealing with objects force one to assume that they are capable of visual rotational invariance, a formal demonstration seems to be lacking (4). An animal model would be useful for the study of the mechanisms underlying these operations, which even robot engineers find cumbersome to implement (5). We now report that pigeons are more efficient than humans at recognizing certain two-dimensional visual patterns regardless of their orientation in the frontal plane.

Ten adult homing pigeons (Columba livia) were maintained at 85 percent of their normal weight throughout the experiment. A Skinner box with three keys was used. Stimuli were presented by projector, the display on the individual keys being controlled by shutters. The forms appeared as white $10-\mathrm{mm}$ by 10 $\mathrm{mm}$ patterns on a dark background 25 $\mathrm{mm}$ in diameter. A computer controlled events within the experimental sessions and recorded the data on a printer (Fig. 1A).

The subjects were trained to master the matching-to-sample discrimination task. Only the final stage of the procedure is described (6). A trial began with the projection of a sample stimulus on the center key. After 15 pecks on it, two comparison stimuli were displayed on the side keys. One comparison form was identical to the sample, and the other was always its mirror image. Half of the subjects were rewarded with a 3-second access to food as soon as they pecked the side key bearing the identical match-
A

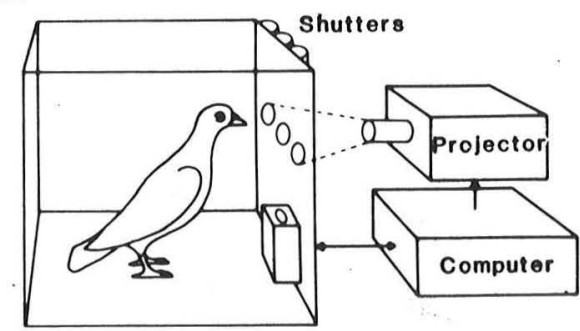

1

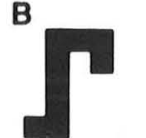

6

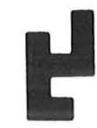

$\theta$

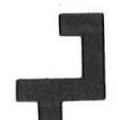

2

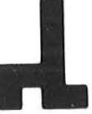

3

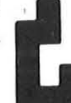

4

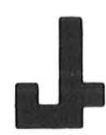

6

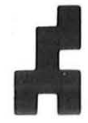

10

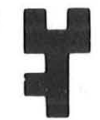

7

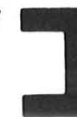

11

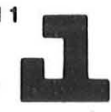

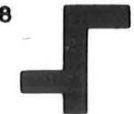

12

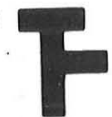

C

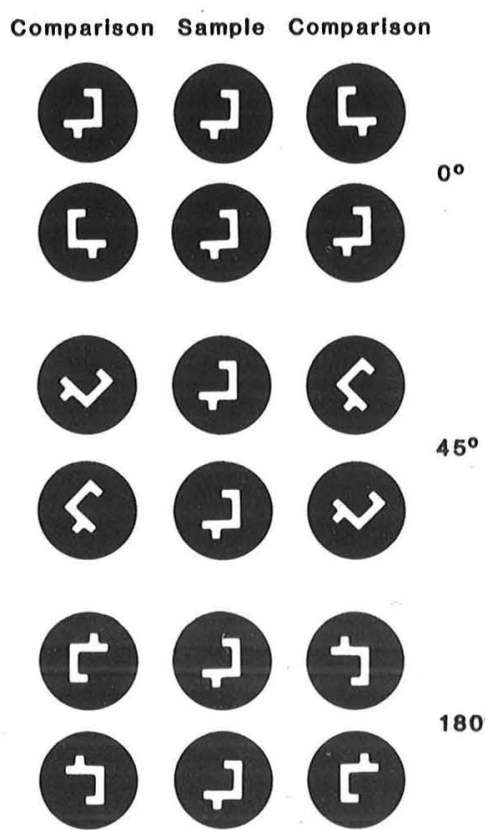

Fig. 1. (A) Experimental apparatus as used with pigeons. (B) Visual forms used. (C) Examples of stimulus sets used for rotational invariance test. 
ing form and punished with 3 seconds of darkness if they pecked the side key bearing the mirror-image, nonmatching form. The reverse contingencies applied for the other subjects. All stimuli were extinguished after a response to a side key. The next trial began after a 15 second interval. If the subject had responded incorrectly in the preceding tri$\mathrm{al}$, the same stimulus set was presented again (correction procedure). If it had responded correctly, the next stimulus set of the sequence was presented. A session ended when 40 correct trials had been completed.

Forty stimulus sets based on forms 1 to 10 (Fig. 1B) were used, half of them with the matching comparison form projected to the right key and half of them to the left. The sequence of stimulus sets was quasi-random (7) and varied from session to session. Training occurred daily until all pigeons consistently yielded $\geq 80$ percent correct responses (some 40 sessions). One subject that did not reach this performance level was rejected. The animals were then habituated to forms presented in nonstandard orientations for ten further sessions. Sixteen sets based on forms 5 and 9 with both the sample and comparison forms rotated by either $45^{\circ}$ or $90^{\circ}$ clockwise were randomly interspersed among 24 normal $0^{\circ}$ training sets.

Testing for rotational invariance began with the introduction of new stimulus sets. The orientation of the comparison forms no longer coincided with that of the sample form, which was henceforth always shown in its normal $0^{\circ}$ orientation (Fig. 1C). The correction procedure was discontinued. The time elapsed between the appearance of the comparison stimuli and the reaction of the subject was recorded for each trial. Otherwise the procedure continued as described above. For the first block of ten sessions, 16 sets based on forms 5 and 9 with the comparison forms rotated by either $45^{\circ}$ and $90^{\circ}$ clockwise were shown randomly inserted among 24 normal training sets. These included eight sets of forms 5 and 9 at $0^{\circ}$ orientation. The next test block incorporated 16 further sets of the same two forms with the comparison forms rotated by $135^{\circ}$ and $180^{\circ}$. Thus all 40 sets shown were based on forms 5 and 9, all five comparison orientations being equally represented. In the third block, the form5 sets were replaced by 20 equivalent sets of form 8 , which the animals had previously experienced only in normal $0^{\circ}$ training sets. The final test dealt with 40 sets based on forms 11 and 12, totally new to the subjects, at all five comparison orientations.
The performance on the novel stimulus configurations, including their first presentation, was well above chance in all four test blocks (Fig. 2) (8). Thus, the animals did not need to learn the new rotation tasks but could rather apply an extant skill.

The chamber was disassembled and only the wall bearing the keys, lights, and feeder was left standing. Twenty-two college and university students, after detailed instruction and ten training trials, faced the same task as the pigeons, except that the intertrial interval was re- duced to 5 seconds and correct responses were rewarded with the equivalent of a 0.5 cent. Stimulus sets based on forms 8 and 11 at all five orientations were presented in two 40-trial sessions. The keys were activated with a hand-held pointer while the subject sat comfortably with the display at reading distance.

Mean reaction times for correct responses and error rates for each form and comparison stimulus orientation within each test block were calculated (Fig. 3). Pigeons and humans were capable of similar accuracy in visual rotation-

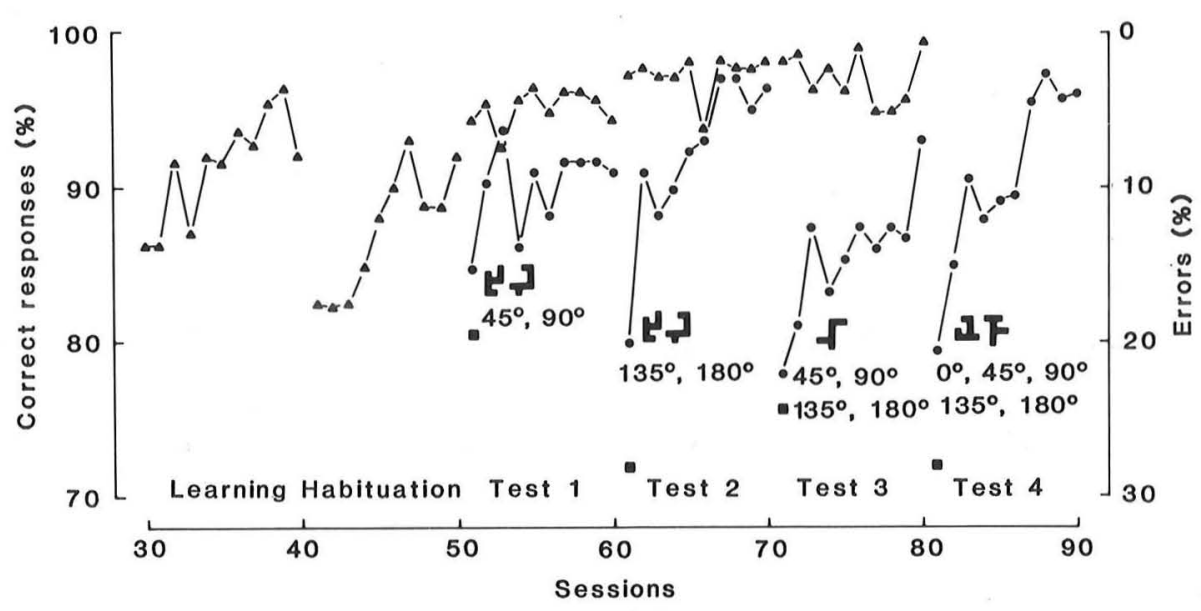

Fig. 2. Mean performance accuracy of pigeons. Symbols: $(\mathbf{\Lambda})$, performance on the training, habituation, and nonnovel stimulus sets; $(\boldsymbol{\Theta})$, performance on the test sets novel to the subjects at the beginning of each test; (回), performance on rotational invariance trials involving the first presentation of the test sets $(8)$.

Fig. 3. Mean reaction times with standard deviations (A) and mean error rates $(\mathrm{B})$ as a function of comparison form rotations. Data are from 9 pigeons and 22 humans. With one exception, combined means corresponding to pairs of forms are shown. Accordingly, results of pigeon tests 1 $(\Delta), 2(\Delta)$, and $4(\mathbf{\square})$ are from 720 trials per comparison orientation; test $3(\diamond), 360$ trials; and the human test $(O), 352$ trials.
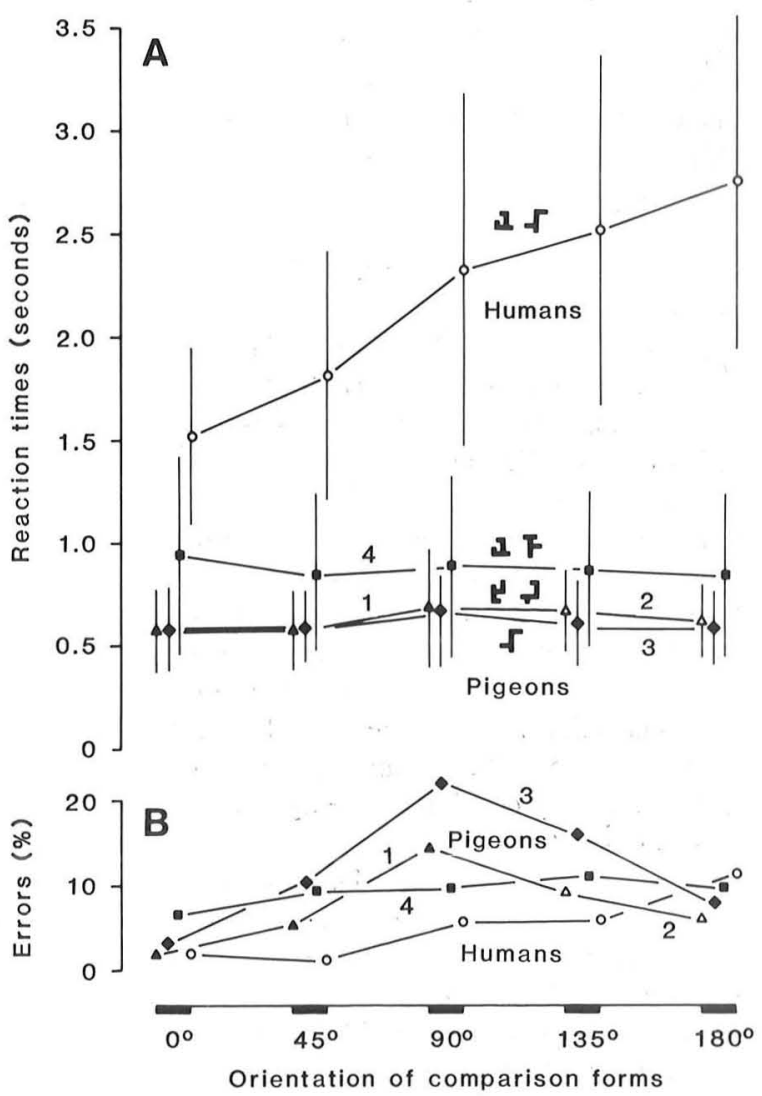
al invariance, judged by the overall error rates (Fig. 3B). The differences may be due to unavoidable instruction, pretraining, and pay-off inequalities. However, the humans generated a reaction-time function that increased monotonically with the angular disparity between the sample and comparison forms, whereas the pigeons produced essentially flat functions (Fig. 3A). Analyses of variance of the pigeon reaction-time data did not show significant orientation effects (all $P$ 's $>.05)$. The human data yielded a significant orientation effect $[F(4,84)=$ 22.76, $P<.001]$ due to a linear trend $[F(1,105)=22.58, P<.001]$.

The human function is in close agreement with those reported by others for similar experiments (3). It conforms to the interpretation that the invariance was achieved by mental rotation, an operation conceived as a serial process in which an engram of a form is rotated until the matching or nonmatching with the percept of another form can be directly assessed (9). Pigeons solved the problem differently and more efficiently, presumably through a parallel mode of information processing (10). The use of mental rotation by humans, however, appears to be almost exclusively restricted to situations involving the discrimination of mirror-image forms. When tasks require the discrimination of distinctly different forms, humans too yield fast and flat functions like those of our pigeons (11). The difference in performance may be ascribed to the possibility that for pigeons, but not for humans, mirror-image forms are as perceptually distinct as any arbitrarily different forms (12).

Although phylogenetically more primitive, pigeons solved a perceptual problem more efficiently than humans. Whether this is related to the primarily midbrain-based visual system of lower vertebrates and the mainly forebrainbased visual system of mammals is not known (13). We believe that the disparity may have originated because of different ecological demands. Pigeons operate visually predominantly on the horizontal plane where the orientation of objects is largely arbitrary, being relative to the position of the observer. Humans primarily view the frontal plane where their orientation and that of objects are highly consistent, being dependent on gravity.

VAlerie D. Hollard JuAN D. Delius

Experimentelle Tierpsychologie, Psychologisches Institut, RuhrUniversität Bochum, D-4630 Bochum, Federal Republic of Germany
References and Notes

1. I. P. Howard and W. B. Templetỏn, Human Spatial Orientation (Wiley, London, 1966), p 294; I. Rock, Orientation and Form (Academic Press, New York, 1973)

2. H. J. Eysenck, Check Your Own I.Q. (Penguin, Harmondsworth, 1974), pp. 109 and 180; W. M. Petrusic et al., Psychol. Res. 40, 139 (1978).

3. R. N. Shepard and J. Metzler, Science 171, 701 (1971); L. A. Cooper and R. N. Shepard, in Visual Information Processing, W. G. Chase, Visual Information Processing, W. G. Chase,
Ed. (Academic Press. New York, 1973), p. 75 S. M. Kosslyn, S. Pinker, G. E. Smith, S. P. S. M. Kosslyn, S. Pinker, G. E. Smith,
Shwartz, Behav. Brain Sci. 2, 535 (1979).

4. G. H. Vetter and E. Hearst, J. Exp. Anal. Behav. 11, 753 (1968); R. J. Herrnstein, D. H Loveland, C. Cable, J. Exp. Psychol. Anim. Behav. Proc. 2, 285 (1976); J. D. Delius and J. Emmerton, in Animal Migration. Navigation. and Orientation, K. Schmidt-Koenig and W. T. Keeton, Eds. (Springer-Verlag, Berlin, 1978), p. 35 .

5. G. G. Dodd and L. Rossol, Eds. Computer Vision and Sensor-Based Robots (Plenum, New York, 1979); A. Rosenfeld and J. S. Weszka, in Digital Pattern Recognition, K. S. Fu, Ed. Digital Pattern Recognition, K. S.
(Springer-Verlag, Berlin, 1980), p. 135

6. The matching-to-sample procedure as used with The matching-to-sample procedure as used with
pigeons has been described [D. E. Carter and T. pigeons has been described [D. E. Carter and T.

7. L. W. Gellermann, J. Genet. Psychol. 42, 206 (1933).

8. The proportions of correct to incorrect responses of the nine pigeons on these first trials were: test 1,29 to 7 ; test 2,26 to 10 ; test 3,28 to 8 ; and test 4,52 to 20 . All ratios were different from chance (binomial tests, $P \leq .01$ ). They also suggest that our pigeons learned an identity or oddity, conceptlike rule IT. R. Zentall, C. A Edwards, B. S. Moore J Exp. Psychol Anim. Behav. Process 7, 70 (1981)) rather than a number of sample specific rules (6).

9. L. A. Cooper, Percept. Psychophys. 19, 296 L. A. Cooper, Percept. Psychophys. 19, 296
(1976); L. A. Cooper and P. Podgorny, J. Exp.

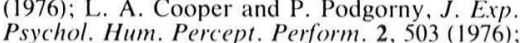
Psychol. Hum. Percept. Perform. 2, 503 (1976):
but see Z. W. Pylyshyn [Mem. Cognit. 7, 19 but see Z. W. Pylyshyn [
10. Videotape recordings showed that the pigeons rarely inclined their heads by more than $30^{\circ}$ and that the head position did not relate in any that the head position did not relate in any obvious way with the orientation of the forms M. C. Corballis, B. A. Nagourney, L. I Shetzer, and G. Stefanatos [Percept. Psy chophys. 24, 263 (1978)] reported that in humans

head orientation is an unimportant variable.

1. O. Aulhorn, Pflügers Arch. Gesamte Physiol Menschen Tiere 250, 12 (1948); D. H. Foster and R. J. Mason, Biol. Cybern. 32, 85 (1979); M. J. White, Bull. Psychon. Soc. 15, 153 (1980); R. D Tilgner and G. Hauske, Z. Exp. Angew. Psychol. (Göttingen) 27, 147 (1980). The reaction times or error functions, though essentially flat. often showed a slight maximum around $90^{\circ}$. Our pigeons may have shown overall an analogous maximum (Fig. 3)

12. M. C. Corballis and I. L. Beale, The Psychology of Left and Right (Erlbaum, Hillsdale, 1976), pp. 29 and 36; M. H. Bornstein, C. G. Gross, J. Z Wolf, Cognition 6, 89 (1978); J. I. Kahn and D. H. Foster, Q. J.Exp. Psychol. 33 A, 155 (1981) Pigeons may still recognize the similarity be tween mirror-symmetric half shapes when recognizing symmetric shapes in a conceptlike way [J. D. Delius and G. Habers, Behav. Biol. 22 336 (1978); J. D. Delius and B. Nowak, Psychol. Res., in press].

13. W. J. H. Nauta and H. J. Karten, in The Neurosciences, Second Study Program, F. O. Schmitt, Ed. (Rockefeller Univ. Press, New York, 1970), p. 7

14.. The work was done while V.D.H was in Bochum with a grant from the Heinrich-Hertz Foundation when on sabbatical leave fromertz Foundation when on sabbatical leave from the Department of Psychology, University of Auck land, New Zealand. Supported by the Research Fund of the Ruhr-Universität and by the Deut sche Forschungsgemeinschaft through its Sonderforschungsbereich 114. We thank P. Kulik C. Werner, the Arbeitsgruppe team and the Werkstatt crew for essential assistance. We are grateful to P. M. Blough, D. S. Blough, M. C. Corballis, G. Reinert, and T. Schulz for comments on an earlier draft. 